\title{
A comparison of nutrition and physical activity behaviors in pregnant women with normal and overweight pre-pregnancy BMI
}

\begin{abstract}
Background: Prenatal counseling by health care professionals on appropriate gestational weight gain, diet and exercise for women who were overweight or obese before pregnancy, are a crucial step towards childhood obesity prevention. This study aims to characterize nutritional behavior change, physical activity and dietary practices differences between women of healthy and overweight pre-pregnancy body mass index (BMI).
\end{abstract}

Methods: Eight pregnant women with healthy pre-pregnancy $\mathrm{BMI}<25 \mathrm{~kg} / \mathrm{m}^{2}$ and five pregnant women with overweight pre-pregnancy $B M I \geq 25 \mathrm{~kg} / \mathrm{m}^{2}$ in their third trimester were recruited through midwives at prenatal appointments to complete a prenatal survey and food frequency questionnaire.

Results: Food frequency questionnaire results revealed that women in the healthy prepregnancy BMI group had greater consumption of vitamin/mineral supplementation, fruits, and vegetables, dairy and herbal supplements. Intake of carbohydrate, dietary fiber, cholesterol, folate, carotene, riboflavin, calcium, and phosphorus, and vitamins A, E, C, B12 intake was greater than the overweight group; however, differences were not statistically significant in food and nutrient intake. The overweight BMI group consumed more saturated fat, discretionary fat, added sugars, non-whole grain, tomato, dark green vegetable servings and caffeine consumption than the women with a healthy BMI but not statistically different. Both groups reported their perceived physical activity level to be the same or somewhat more active compared to other pregnant women of similar age.

Conclusions: Pre-pregnancy $\mathrm{BMI} \geq 25 \mathrm{~kg} / \mathrm{m}^{2}$ women were less likely to follow recommendations from health care professionals or friends/relatives and could benefit from nutrition education and intervention.

Keywords: prenatal nutrition education, prenatal dietary intake, pre-pregnancy BMI, overweight, prevention, demographic
Volume 5 Issue 3 - 2016

\author{
Rose Ann Mathai,' Esmail Bonakdarian, ${ }^{2}$ \\ Juhee Kim, ${ }^{3}$ Leila Shinn, ${ }^{4}$ Hannah Holscher, ${ }^{4}$ \\ Kelly Swanson ${ }^{5}$ \\ 'Nutrition Science Department, Dominican University, USA \\ ${ }^{2}$ Department of Computer Science, Dominican University, USA \\ ${ }^{3}$ Department of Public Health, East Carolina University, USA \\ ${ }^{4}$ Department of Food Science and Human Nutrition, University \\ of Illinois Urbana-Champaign, USA \\ ${ }^{5}$ Department of Animal Sciences, University of Illinois Urbana- \\ Champaign, USA
}

\begin{abstract}
Correspondence: Rose Ann Mathai PhD RD, Assistant Professor, Director, Didactic Program in Dietetics, Nutrition Science Department, Dominican University, 7900 W. Division St, River Forest, IL 60305, Tel (708)5246906, Fax (708) 4885 I I7, Email rmathai@dom.edu
\end{abstract}

Received: September 27, 2016 | Published: November 22 2016

\section{Introduction}

In the United States, approximately two third of women are overweight and one third obese prior to becoming pregnant ${ }^{1}$ and approximately half of them experience excess gestational weight gain. ${ }^{2}$ Women entering pregnancy as obese are at a greater risk for developing insulin resistance and gestational diabetes. ${ }^{1}$ Excess maternal weight gain during pregnancy beyond the $25-35$ pounds recommended for a woman with healthy body mass index (BMI) can increase the risk for prenatal hypertensive disorders, labor and delivery complications, and breastfeeding challenges. ${ }^{1}$ In addition, postpartum weight retention increases a woman's future risk for obesity, cardiovascular disease, and type 2 diabetes. ${ }^{3,4}$ The consequences of maternal obesity on the child include large birth weight and increased risk of obesity, insulin resistance, metabolic disorders, impaired cognitive function and behavior later in life. ${ }^{1,5}$

Prevention of excess gestational weight gain may be achieved with a screening tool prior to or during early pregnancy. Screening women for unhealthy dietary patterns can be a useful step for implementing changes in food choices early in pregnancy. The importance of dietary education during pregnancy is reflected by a study that found that $72 \%$ of pregnant women who had an 'unhealthy' dietary pattern early in pregnancy continued to eat the same way in their second trimester and 57\% in the third trimester. ${ }^{6,7}$ Food choices were shown to effectively change in pregnant women when they were provided with a combination of dietary counseling and healthy food products, as evidenced by increases in the number of servings of vegetables, fruits, soft margarines and vegetable oils and decreases in butter consumption. ${ }^{8}$ As a result, dietary intake significantly improved by increasing nutrients such as polyunsaturated fatty acids, dietary fiber, vitamin $\mathrm{E}$, folate and ascorbic acid intake and decreasing saturated fatty acid intake. ${ }^{8}$ The combination of dietary counseling and administration of healthy food products has the potential to promote a healthy weight gain if continued throughout the pregnancy.

Another approach to promoting healthy weight gain during pregnancy is to provide physical activity or physical activity combined with nutrition counseling. ${ }^{9}$ Pregnant women who discuss exercise with their obstetricians are more likely to take part in regular exercise, which can lead to decreased gestational weight gain, better muscle tone, lower resting heart rate in baby, leaner children that perform better in academics and athletics compared to non-exercising counterparts. ${ }^{10}$ A customized nutrition and exercise lifestyle regime has been shown to effectively prevent excess gestational weight gain for pregnant women with a pre-pregnancy BMI of $\geq 25 \mathrm{~kg} / \mathrm{m}^{2}{ }^{11}$

Counseling pregnant women who are overweight or obese before pregnancy on appropriate gestational weight gain and postpartum weight loss represents an important area for childhood obesity 
prevention. $^{2}$ Despite the significant impact this counseling may provide, no study has examined the effect of nutrition and physical activity prenatal counseling strategies on gestational weight gain in women with healthy pre-pregnancy BMIs and compared potential differences. The current study fills the gap in the literature by addressing the following research question: How do food choices, physical activity and dietary intake differ between healthy weight $\left(\mathrm{BMI}<25 \mathrm{~kg} / \mathrm{m}^{2}\right)$ and overweight pregnant women $\left(\mathrm{BMI} \geq 25 \mathrm{~kg} / \mathrm{m}^{2}\right)$ based on their pre-pregnancy BMI?

We hypothesize that women with a healthy pre-pregnancy BMI $<25 \mathrm{~kg} / \mathrm{m}^{2}$ make food choices that adhere to pregnancy recommendations and have a dietary intake lower in fat and higher in fruits and vegetables as well as higher physical activity level when compared to women with an overweight/obese pre-pregnancy $\mathrm{BMI} \geq 25 \mathrm{~kg} / \mathrm{m}^{2}$. These improvements in food choices, physical activity levels and dietary intake could affect gestational weight gain and lead to improvements in the overall health of the mother and child.

\section{Methods}

This pilot project aimed to determine nutritional and physical activity practices and attitudes of pregnant women. Thirteen pregnant women in their third trimester of pregnancy were recruited by midwives at their prenatal appointments in Urbana, IL and given informational flyers at the Obstetrics and Gynecology (OB GYN) office by midwives during a prenatal visit. OB GYN midwives briefly described the study and interested women were asked to complete a reply card with their name, due date, and contact information to be placed in a drop box in the OB GYN office. Members of the research staff picked up the reply cards from the drop box in the OB GYN office and contacted the women by telephone or email. Research staff explained the study in full detail and obtained verbal consent. After receiving verbal consent, women were sent a study packet containing a written consent to be signed along with a pregnancy survey and a food frequency questionnaire.

To be included in this study, women had to have a healthy pregnancy without medical complications. They also had to deliver vaginally and plan to breastfeed their baby for a period of time after birth (greater than 1 week), followed by introduction of formula. Women were excluded from the study if they smoked during their pregnancy, or had any serious medical conditions during their pregnancy. In addition, the infant should not have any chronic medical conditions and not taking any regular medications.

\section{Demographic measures}

The prenatal survey was administered to collect demographic information as shown in Tables $1 \& 2$ as well as gather information about the woman's perception of health consciousness.

\section{Weight Status}

Self-reported pre-pregnancy weight ${ }^{12}$ and height from the prenatal survey were used to calculate the pre-pregnancy body mass index $\left(\mathrm{BMI}=\left(\right.\right.$ weight $(\mathrm{lb}) /(\text { height(in) })^{2 * 703)}$. BMI was used to categorize participants into healthy weight $\left(\mathrm{BMI}<25 \mathrm{~kg} / \mathrm{m}^{2}\right)$ and overweight groups (BMI $\geq 25 \mathrm{~kg} / \mathrm{m}^{2}$ ). Accordingly, we may subsequently refer to participants with $\mathrm{BMI}<25 \mathrm{~kg} / \mathrm{m}^{2}$ as the "healthy" group and those with $\mathrm{BMI} \geq 25 \mathrm{~kg} / \mathrm{m}^{2}$ as "overweight".

Current weight was also used to calculate pregnancy weight gain, which was compared to recommended pregnancy weight gain guidelines from the Institute of Medicine based on pre-pregnancy BMI.

\section{Nutritional behavior changes}

Our questions shown in Table 3 were drawn from a validated survey used in the Infant feeding practices study II (IFPS II) conducted in 2005-2007 by the Centre for Disease Control (CDC) and Food and Drug Administration (FDA). ${ }^{12}$

\section{Dietary intake and nutrient intake}

A food frequency questionnaire was used to assess the diet during pregnancy. The National Health and Nutrition Examination Survey (NHANES) Food Questionnaire is a validated survey instrument developed by the National Cancer Institute (NCI) that measures the frequency of consumption in various food groups without asking about portion sizes. Data were analyzed using NCI Diet*Calc program to generate daily food frequency estimates and nutrient intake, shown in Table $4 \& 5$, respectively. ${ }^{13}$

\section{Physical activity}

Physical activity was measured using the following questions:1) "Compared with other pregnant women of your age, would you consider yourself to be a) much more active b) somewhat active c) about the same d) somewhat less active e) much less active" and 2) "Currently, at least once a week do you engage in any regular activity like brisk walking, gardening, housework, jogging, cycling, etc., (long enough to work up a sweat)? Yes/No. If yes, how many hours per week? These questions were validated and taken from the Avon Longitudinal Study of Parents and Children (ALSPAC) study. ${ }^{14}$

\section{Statistical analysis}

The data was analyzed using the open source statistical computing $\mathrm{R}$ environment (Version 3.1.1, 2014, http://www.r-project.org/). Descriptive statistics, such as means, standard deviations, range and various box plots were generated for the data. Parametric and nonparametric tests (using Wilcoxon) for differences in the mean were performed for the demographic, as well as the food and nutrition data. A p value $<.05$ was used to denote statistical significance.

\section{Results}

Table 1 shows significant differences in the pre-pregnancy BMI, pre-pregnancy and current pregnancy weight between healthy and overweight groups. No other statistically significant differences were detected with the remaining demographic variables. Mothers were in their late twenties and recruited during the last month of pregnancy. Fathers worked approximately 40 hours/week and mothers approximately 27 hours/week. Women in the healthy group lived in their home one year longer than the overweight group on average.

There was no significant difference in weight gain during pregnancy between the two groups. Of the 13 participants, over half fell into the normal range regarding pre-pregnancy BMI (Between $18.5-24.9 \mathrm{~kg} / \mathrm{m}^{2}$ ). One participant was underweight, four were overweight, and one was classified as obese. Of the mothers with prepregnancy healthy weight group, 3 out of 8 gained excess amount of weight during pregnancy, compared to 3 out of 5 women in the overweight group who gained excess weight according to the Institute of Medicine (IOM) weight gain guidelines. Five women in the healthy weight group and three in the overweight group reported satisfaction with their current pregnancy body weight (data not shown). 
Table I Mothers demographic information

\begin{tabular}{|c|c|c|c|c|c|c|}
\hline & \multicolumn{2}{|c|}{ All $(n=13)$} & \multicolumn{2}{|c|}{$B M I<25(n=8)$} & \multicolumn{2}{|c|}{$B M I \geq 25(n=5)$} \\
\hline & Mean & SD & Mean & SD & Mean & SD \\
\hline Mothers Age (years) & 29.82 & 2.79 & 30.29 & 3.73 & 29 & 4.32 \\
\hline Child Birth Order & 2.39 & 0.87 & 2.63 & 1.06 & 2 & 0 \\
\hline Weeks Pregnant & 37.62 & 1.98 & 38.5 & 1.77 & 36.2 & 1.48 \\
\hline Length of Home Residency (months) & 37.39 & 20.89 & 43.38 & 16.62 & 27.8 & 25.27 \\
\hline Household Size (number of people) & 3.46 & 1.13 & 3.5 & 1.52 & 3.4 & 0.55 \\
\hline Mothers Work Per Week (hours) & 26.71 & 15.56 & 27.33 & 15.54 & 26.25 & 17.97 \\
\hline Fathers Work Per Week (hours) & 40.58 & 9.41 & 42.43 & 3.82 & 38 & $|4.4|$ \\
\hline Height (Inches) & 65.39 & 2.76 & 65.75 & 2.19 & 64.8 & 3.7 \\
\hline Pre-Pregnancy Weight* (lbs) & $|43.3|$ & 24.91 & 129.5 & 11.07 & 165.4 & 25.61 \\
\hline Current Pregnancy Weight† (lbs) & 177.15 & 27.49 & 163.75 & 13.28 & 198.6 & 32.01 \\
\hline Pre-pregnancy BMI $\ddagger\left(\mathrm{kg} / \mathrm{m}^{2}\right)$ & 23.58 & 3.85 & 21.08 & 1.84 & 27.58 & 2.46 \\
\hline Weight Gain during Pregnancy (lbs) & 33.85 & 9.71 & 34.25 & 8.05 & 33.2 & 12.99 \\
\hline
\end{tabular}

${ }^{*}$ p-value $=.0295$

tp-value $=.045 \mathrm{I}$

$\neq \mathrm{p}$-value $=.0016$

P-value $<0.05$ considered significant, $\mathrm{P}$-value computed by Wilcoxon test

Table 2 shows 12 out of 13 participants had an annual income of over $\$ 25,000$ and more than half had earned a four year college degree or beyond. Most of the participants felt that they were about the same or somewhat more active and perceived themselves to be somewhat or the same level of health consciousness as others

Table 2 Maternal income, education and activity level. ${ }^{1-3}$

\begin{tabular}{|c|c|c|c|}
\hline Income & All & BMI $<25$ & $\mathbf{B M I} \geq \mathbf{2 5}$ \\
\hline Between $\$ 15,000-\$ 24,999$ & 1 & 1 & 0 \\
\hline Between $\$ 25,000$ - $\$ 34,999$ & 2 & 0 & 2 \\
\hline Between $\$ 35,000-\$ 49,999$ & 3 & 3 & 0 \\
\hline Between $\$ 50,000$ - $\$ 74,999$ & 2 & I & $\mathrm{I}$ \\
\hline Between $\$ 75,000$ - $\$ 99,999$ & 2 & 2 & 0 \\
\hline Over $\$ 100,000$ & 3 & 1 & 2 \\
\hline \multicolumn{4}{|l|}{ Education } \\
\hline High school/GED & 4 & 2 & 2 \\
\hline Some college & 1 & 0 & 1 \\
\hline 2 year college (Associate) & 1 & I & 0 \\
\hline 4 year college & 3 & 3 & 0 \\
\hline Master's degree & 2 & 2 & 0 \\
\hline Doctorate degree & 2 & 0 & 2 \\
\hline \multicolumn{4}{|l|}{ Level of Activity ${ }^{4}$} \\
\hline Somewhat active & 5 & I & 4 \\
\hline About the same & 7 & 6 & 1 \\
\hline Somewhat less active & I & I & 0 \\
\hline
\end{tabular}




\begin{tabular}{llll} 
Table Continued.... & All & BMI $<\mathbf{2 5}$ & BMI $\geq \mathbf{2 5}$ \\
\hline Income & & & \\
\hline Health Consciousness $^{5}$ & 2 & $\mathrm{I}$ & $\mathrm{I}$ \\
\hline Much more health conscious & 7 & 5 & 2 \\
Somewhat health conscious & 4 & 2 & 2 \\
\hline
\end{tabular}

'Numbers shown in the table represent the frequency

${ }^{2} \mathrm{BMI}$ in $\mathrm{kg} / \mathrm{m}^{2}$

${ }^{3}$ No statistical significance detected

${ }^{4}$ Level of activity was surveyed in comparison to other pregnant women of similar age

${ }^{5}$ Degree of health consciousness was surveyed in comparison to other pregnant women of similar age

When asked about regular physical activity per week, there was no statistical significance between the groups (data not shown). Five women indicated that they did not exercise, four from the healthy weight group and one from the overweight group. The eight women that participated in regular physical activity were evenly split between the two groups. The average number of hours per week engaged in physical activity was 3 hours for women in the healthy weight group and 6.25hours for the overweight group.

The number of women that chose to change their nutritional behavior to adhere to pregnancy nutrition recommendations as a result of friend/relative or health professional guidance is shown in Table 3. There were no significant differences between the two groups. Of the recommendations made by health care professionals, the one to decrease lunch meat had the highest compliance rate in both groups followed by lunch meat recommended by their friend/relative. Other recommended changes that were adhered to by both groups included decreasing alcohol, canned tuna and shellfish. Interestingly, the recommended increases in vitamin/mineral supplementation, fruits, and vegetables, dairy and herbal supplements were found only in women in the healthy weight group.

Table 3 Number of women that made nutritional behavior changes during pregnancy as a result of friend/relative or health professional recommendations. ${ }^{1-3}$

\begin{tabular}{|c|c|c|c|}
\hline Eating less of this food-recommended by friend/relative (FR) or health professional (HP) & All & BMI $<25$ & $\mathbf{B M I} \geq \mathbf{2 5}$ \\
\hline Canned tuna - HP & 2 & I & I \\
\hline Swordfish, shark, tilefish, or king mackerel - HP & I & 0 & I \\
\hline Shellfish - HP & 2 & I & I \\
\hline Luncheon meats - HP & 4 & 3 & 1 \\
\hline Luncheon meats - FR & 2 & I & I \\
\hline Alcoholic drinks - HP & 2 & I & I \\
\hline Alcoholic drinks - FR & 1 & I & 0 \\
\hline Vegetables - FR & I & 0 & I \\
\hline Fruits - FR & I & 0 & I \\
\hline Soft drinks - HP & I & I & 0 \\
\hline Eating more of this food-recommended by friend/relative (FR) or health professional (HP) & All & BMI $<25$ & $\mathbf{B M I} \geq \mathbf{2 5}$ \\
\hline Milk or other dairy foods - HP & I & 0 & I \\
\hline Vitamins or mineral supplement - HP & 3 & 3 & 0 \\
\hline Vitamins or mineral supplement - FR & 2 & 2 & 0 \\
\hline Any herbal or botanical supplement - FR & I & I & 0 \\
\hline Vegetables - HP & I & I & 0 \\
\hline Vegetables - FR & $\mathrm{I}$ & I & 0 \\
\hline Fruits - HP & I & I & 0 \\
\hline Fruits - FR & I & I & 0 \\
\hline
\end{tabular}

'Numbers shown in the table represent the frequency

${ }^{2} \mathrm{BMI}$ in $\mathrm{kg} / \mathrm{m}^{2}$

${ }^{3}$ No statistical significance detected. 
Table 4 shows women with pre-pregnancy in the healthy weight group consumed a greater number of servings of whole grains, vegetables, dry bean and peas, total vegetable, deep yellow vegetable, white potato, total fruit, citrus melon berry, milk, dairy, cheese servings although not statistically different from the overweight group. Table 5 indicates women with a pre-pregnancy $\mathrm{BMI}<25$ had a higher intake of carbohydrate, dietary fiber, cholesterol, vitamin A, E, C, B12, folate, carotene, riboflavin, calcium and phosphorus and less saturated fat although not statistically different from the BMI $\geq 25$ group . Women in the overweight group showed greater consumption of discretionary fat, added sugars and caffeine intake than their counterparts but not statistically different.

Table 4 Food consumption patterns. ${ }^{1,2}$

\begin{tabular}{|c|c|c|c|c|c|c|}
\hline \multirow[b]{2}{*}{ Food servings/day } & \multicolumn{2}{|c|}{ All $(n=13)$} & \multicolumn{2}{|c|}{$B M I<25(n=8)$} & \multicolumn{2}{|c|}{$B M I \geq 25(n=5)$} \\
\hline & Mean & SD & Mean & SD & Mean & SD \\
\hline Total number of grain servings & 4.623 & 2.206 & 4.673 & 1.939 & 4.544 & 2.828 \\
\hline Number of whole grain servings & 0.958 & 0.582 & I.07I & 0.618 & 0.776 & 0.532 \\
\hline Number of non whole grain servings & 3.654 & 1.742 & 3.59 & 1.435 & 3.756 & $234 \%$ \\
\hline Total number of vegetable servings & 2.884 & I & 3.026 & 1.078 & 2.656 & 0.929 \\
\hline Number of dark green vegetable servings & 0.39 & 0.32 & 0.378 & 0.371 & $0.4 I$ & 0.254 \\
\hline Number of deep yellow vegetable servings & 0.253 & 0.16 & 0.278 & 0.19 & 0.214 & 0.103 \\
\hline Number of dry bean and pea servings & 0.152 & 0.15 & 0.204 & 0.171 & 0.07 & 0.047 \\
\hline Number of white potato servings & 0.516 & 0.309 & 0.546 & 0.338 & 0.468 & 0.286 \\
\hline Number other starchy vegetable servings & 0.24 & 0.173 & 0.253 & 0.175 & 0.22 & 0.189 \\
\hline Number of tomato servings & 0.435 & 0.238 & 0.369 & 0.119 & 0.542 & 0.35 \\
\hline Number of other vegetable servings & 0.895 & 0.343 & 0.995 & 0.395 & 0.734 & 0.165 \\
\hline Total number of fruit servings & 2.482 & 1.101 & 2.741 & 1.131 & 2.068 & 1.025 \\
\hline Number of citrus melon berry servings & 0.868 & 0.494 & 0.93 & 0.51 & 0.77 & 0.508 \\
\hline Number of other fruit servings & 1.612 & 0.863 & 1.806 & 0.986 & 1.3 & 0.578 \\
\hline Total number of dairy servings & 2.462 & 2.073 & 2.726 & 2.037 & 2.038 & 2.295 \\
\hline Number of milk servings & 1.783 & 2.076 & 1.931 & 2.01 & 1.546 & 2.397 \\
\hline Number of yogurt servings & 0.146 & 0.185 & 0.19 & 0.228 & 0.076 & 0.049 \\
\hline Number of cheese servings & 0.523 & 0.232 & 0.591 & 0.187 & 0.414 & 0.276 \\
\hline Grams of discretionary fat & 61.311 & 16.34 & 59.905 & 15.815 & 63.56 & 18.789 \\
\hline Teaspoons of added sugars & 13.659 & 7.34 & 13.36 & 5.409 & 14.138 & 10.487 \\
\hline
\end{tabular}

'BMI in $\mathrm{kg} / \mathrm{m}^{2}$

${ }^{2}$ No statistical significance detected.

Table 5 Daily nutrient intake patterns and its comparison to the Dietary Reference Intake (DRI).,2

\begin{tabular}{|c|c|c|c|c|c|c|c|}
\hline \multirow[b]{2}{*}{ DRI } & \multirow[b]{2}{*}{ Nutrient } & \multicolumn{2}{|c|}{ All $(n=13)$} & \multicolumn{2}{|c|}{ BMI $<25(n=8)$} & \multicolumn{2}{|c|}{ BMI $\geq 25(n=5)$} \\
\hline & & Mean & SD & Mean & SD & Mean & SD \\
\hline & Energy (kcal) & 1800.32 & 376.72 & 1837.86 & 264.72 & 1740.24 & 543.86 \\
\hline \multirow[t]{5}{*}{71} & Protein $(g)$ & 68.87 & 20.36 & 71.95 & 20.37 & 63.93 & 21.63 \\
\hline & Total fat (g) & 74.13 & 17.96 & 73.22 & 16.55 & 75.6 & 22.01 \\
\hline & Saturated fat (g) & 24.72 & 5.73 & 25.06 & 5.23 & 24.18 & 7.07 \\
\hline & Monounsaturated fat (g) & 29.45 & 9.34 & 29.26 & 8.46 & 29.76 & 11.68 \\
\hline & Polyunsaturated fat (g) & 14.72 & 3.65 & 13.6 & 3.41 & 16.5 & 3.63 \\
\hline \multirow[t]{2}{*}{175} & Cholesterol (mg) & 186.67 & 63.73 & 198.18 & 72.74 & 168.25 & 47.3 \\
\hline & Carbohydrate (g) & 221.55 & 58.75 & 229.07 & 36 & 209.52 & 88.28 \\
\hline
\end{tabular}


Table Continued....

\begin{tabular}{|c|c|c|c|c|c|c|c|}
\hline \multirow[b]{2}{*}{ DRI } & \multirow[b]{2}{*}{ Nutrient } & \multicolumn{2}{|c|}{ All $(n=13)$} & \multicolumn{2}{|c|}{ BMI < $25(n=8)$} & \multicolumn{2}{|c|}{$B M I \geq 25(n=5)$} \\
\hline & & Mean & SD & Mean & SD & Mean & SD \\
\hline \multirow[t]{2}{*}{28} & Dietary fiber (g) & 15.77 & 3.66 & 16.83 & 3.81 & 14.07 & 3 \\
\hline & Alcohol (g) & 1.83 & $2.7 I$ & 2.5 & 3.28 & 0.75 & 0.88 \\
\hline 550 & Vitamin A (mcg) & 1116.4 & 379.19 & 1185.62 & $4|5.7|$ & 1005.64 & 322.51 \\
\hline 12 & Vitamin E (mg) & 8.82 & 2.3 & 8.57 & 2.24 & 9.22 & 2.59 \\
\hline 70 & Vitamin C (mg) & 106.18 & 40.75 & 108.66 & 37.77 & $|02.2|$ & 49.52 \\
\hline 1.2 & Thiamin (mg) & 1.25 & 0.28 & 1.28 & 0.19 & 1.2 & 0.41 \\
\hline 1.2 & Riboflavin (mg) & 1.87 & 0.79 & 1.96 & 0.76 & 1.73 & 0.89 \\
\hline 14 & Niacin (mg) & 16.86 & 5.35 & 16.28 & 3.25 & 17.8 & 8.1 \\
\hline 1.6 & Vitamin B6 (mg) & 1.53 & 0.29 & $\mathrm{I} .54$ & 0.27 & 1.51 & 0.35 \\
\hline 520 & Folate (mcg) & 289.98 & 49.38 & 294.13 & 25.22 & 283.32 & 78.18 \\
\hline 2.2 & Vitamin BI2 (mcg) & 3.69 & 1.99 & 4.07 & 2.08 & 3.09 & 1.89 \\
\hline 800 & Calcium (mg) & 1024.99 & 591.74 & 1116.9 & 582.45 & 877.92 & 642.54 \\
\hline 580 & Phosphorus (mg) & 1277.76 & 453.92 & 1364.76 & 464.05 & I I38.56 & 449.35 \\
\hline 290 & Magnesium (mg) & 285.77 & 52.8 & 294.75 & 45.53 & 271.41 & 65.7 \\
\hline 22 & Iron (mg) & 10.92 & 2.44 & 11.05 & 1.06 & 10.7 & 3.98 \\
\hline 9.5 & Zinc (mg) & 9.47 & 2.33 & 9.97 & 2.54 & 8.67 & 1.92 \\
\hline 0.8 & Copper (mg) & 1.16 & 0.22 & 1.19 & 0.18 & I.I & 0.3 \\
\hline 1500 & Sodium (mg) & 2614.69 & 648.29 & 2634.75 & 533.21 & 2582.58 & 872.47 \\
\hline \multirow[t]{2}{*}{4700} & Potassium (mg) & 2821.14 & 776.28 & 2958.38 & 732.22 & 2601.56 & 878.42 \\
\hline & Caffeine (mg) & $130.2 \mid$ & 203.59 & 73.84 & 100.29 & 220.41 & 300.36 \\
\hline
\end{tabular}

'BMI in $\mathrm{kg} / \mathrm{m}^{2}$

${ }^{2}$ No statistical significance detected.

\section{Discussion}

This pilot study characterized maternal food choices, physical activity and dietary intake patterns in a cohort of pregnant women participating in a pilot study. Our comparison between the healthy and overweight groups did not show significant differences in dietary intake, physical activity level, and gestational weight gain. Interestingly, a greater proportion of women with healthy weights chose to make food choices according to pregnancy recommendations as advised by both health care professionals and friends or relatives. These changes included consuming less luncheon meat and more vitamin or mineral supplements, vegetables, and fruits.

The results of this study suggested that women in the healthy weight group exhibited positive dietary changes with numerically larger, yet non-significant number of servings of whole grains, vegetables, dry bean and peas, vegetable, fruit, and dairy servings compared to their overweight counterparts. In comparison to the Academy of Nutrition and Dietetics (AND) prenatal dietary guidelines, all participants in our study were lacking the number of whole grain and dairy servings, but the healthy weight group met the fruit and vegetable recommendations. ${ }^{15}$

Women in the overweight group were less likely to make food choices in line with pregnancy recommendations from health care professionals or friend/relative, although not statistically significant.
The overweight group had higher levels of discretionary fat, added sugars and caffeine consumption than the women from the healthy weight group. Women in the healthy weight group consumed numerically more carbohydrate, dietary fiber, cholesterol, vitamin A, E, C, B12, folate, carotene, riboflavin, calcium and phosphorus and less saturated fat than their overweight counterpart, as shown in Table 5. In addition, the overweight group consumed greater than $200 \mathrm{mg}$ of caffeine/day, a level that was higher than the healthy weight group. Excessive caffeine consumption is correlated to a higher risk of both early and late miscarriage. ${ }^{16}$

Previous evidence reveals that lower maternal education, younger age, greater BMI and ethnicity are correlated with unhealthy eating behaviors in pregnant women. ${ }^{6,7}$ In our study, there was no significant difference in education, age or ethnicity to account for the variability in eating behaviors between the groups. The study sample is a somewhat 'health conscious' population since they plan to breastfeed and maintain healthy pregnancy without any pregnancy complications. A numerically greater percentage of pregnant woman with pre-pregnancy $\mathrm{BMI}<25$ classified themselves as 'somewhat health conscious' as well as 'about the same' physical activity level when compared to other pregnant women of similar age. Consistent with previous studies, approximately one third of these women gained excess weight beyond the recommended weight gain guidelines with a greater percentage gaining excess weight in the $\mathrm{BMI} \geq 25$ group. 
The positive dietary changes and perceived health consciousness in the women in the healthy weight group could be due to the higher education level. There were a larger percentage of women with 4year college degrees and Master's degrees in the healthy group compared to the overweight group. It is also possible that women of the healthy weight group were already following a healthy lifestyle with diet and exercise and making positive dietary changes based on advice from their primary care physician rather than the prenatal team of health professionals. All study participants received prenatal care from the same group of midwives and presumably had the same level of counseling. Income differences between the subjects can be a factor in their access to healthy foods. There could be other barriers to engaging in a healthy lifestyle such as lack of knowledge or social and family support. Potential differences could exist between the women in their level of engagement in their own pregnancy. Women in the healthy weight group lived a larger number of months at their current residences, which suggests a greater level of social stability.

This study supports findings from De Jersey et al., ${ }^{17}$ who reported that overweight women appear less likely to meet recommendations for diet and weight gain during pregnancy. ${ }^{17}$ Yet, the present study adds new information by reporting more detailed food and nutrient intake comparisons between the two groups studied. This finding draws attention to the need for improved nutrition education and behavioral modification techniques to support a healthy lifestyle and prevent excess weight gain during pregnancy. Individualized dietary and exercise screening and counseling can change maternal dietary and physical activity behavior and yield improved results for maternal and child health. ${ }^{6,8,10}$ Currently, prenatal nutrition counseling and dietary assessment services are not uniformly available and may not be covered by all medical insurance plans. ${ }^{1}$ The cost for implementing these programs widely is a challenge that has yet to be solved, though the need is evident. Health care professionals provide the greatest opportunity for education and support towards behavior change to improve diet and physical activity during pregnancy for this population.

In a trial of obese women with a high risk of gestational diabetes mellitus, subjects were provided with regular, individual prenatal nutrition counseling, and physical activity sessions. ${ }^{1}$ Another trial monitored weight gain of obese pregnant women by health care professionals and sent educational materials to patients through the mail. Both trials showed minor improvement on the subjects' dietary habits but no effect on gestational weight gain, birth weight, the rate of cesarean delivery, or postpartum weight retention. Successful intervention strategies to lower gestational weight gain in other studies included additional visits with midwives to educate and encourage women to change their behavior, invitations to fitness classes, 10 onehour dietary consultations designed to assist in gestational weight gain restriction, and specific individualized counseling for obese pregnant women. ${ }^{1}$

The present study had limitations. First, the study was limited by a small sample size, as it was a pilot study to characterize nutritional behavior change, physical activity and dietary practices between women of healthy and overweight pre-pregnancy BMI. Sample selection was not calculated using a statistical tool. The small sample size limited our ability to draw many statistical conclusions. It would be of interest to use a larger sample size to compare nutritional behavior change, physical activity and dietary practices differences between women of healthy and overweight pre-pregnancy BMI. It would also be of interest to assess whether individuals from various socioeconomic and cultural backgrounds account from some of the differences in these women. Second, it is possible that some of the survey questions and food questionnaire may have been misinterpreted by the study participants. Third, the prenatal survey is not a validated tool from other studies. Fourth, BMI was computed based on self-reported pre-pregnancy weight. BMI is also not an accurate measure of body fat since BMI is a reflection of the sum of lean body mass, fat mass and body water in proportion to height. Fifth, health care professionals were not surveyed to assess the extent of prenatal dietary counseling and quality of advice. Therefore, the interpretation of Table 3 is not based on any information received from the health care professionals providing prenatal care and their specific recommendations. Furthermore, nutrition recommendations from a prenatal health care professional would be different compared to formal dietary counseling by a registered dietitian. Our study sample most likely did not see a registered dietitian since they had to have a healthy pregnancy without medical complications in order to be included in the study. Regardless of the nature of nutrition intervention received, we know that our study sample was somewhat health conscious because some women in our study sample chose to make changes in their food choices based on pregnancy and is reflected in the dietary and nutrient intake data collected in this study.

Strength of our study is that we used the NHANES Food Questionnaire, which is a validated tool of dietary intake. In addition, the measures used for nutrition behavior change and physical activity were used from validated sources from the IFPS II and ALSPAC studies. All of the pregnant women in this study received the same nutritional and physical education prenatal counseling, since they were cared for by the same group of midwives at the same hospital, which eliminates the variation between the health providers.

\section{Conclusion}

This study provides more information on how adherence to nutritional counseling, physical activity and dietary practices differ between women of healthy and overweight pre-pregnancy BMI levels. Overweight women were less likely to make nutritional behavior changes during pregnancy based on the recommendations from health care professionals or friend/relatives. Physical activity levels did not differ between groups. However, women in the healthy weight group consumed a higher quality diet consisting of whole grains, vegetables, legumes, potato, total fruit, and dairy servings compared to the overweight group. Our results support the need for more individualized diet and exercise counseling in pregnant women, especially those with a pre-pregnancy $\mathrm{BMI} \geq 25 \mathrm{~kg} / \mathrm{m}^{2}$. Improvements to the level of prenatal education and behavior counseling to achieve a healthy lifestyle may have a significant impact on the overall health of mothers from all different weight statuses.

\section{Acknowledgements}

The authors would like to thank the Carle Hospital midwives staff for their cooperation with this research. Without their help, this research would not have been made possible The authors would also like to acknowledge nutrition student, Leah Johnston, for her unwavering assistance in this study and to Dr. Dipti Dev for her valuable editorial assistance.

\section{Funding}

This work was in part supported by the Hatch Project \# ILLU971-350.

\section{Conflict of interest}

The author declares no conflict of interest. 


\section{References}

1. Rasmussen KM, Yaktine AL. Institute of Medicine (US), National Research Council (US) Committee to Reexamine IOM Pregnancy Weight Guidelines. Weight Gain during Pregnancy: Reexamining the Guidelines. USA: National Academies Press; 2009.

2. Heerman WJ, Bian A, Shintani A, et al. Interaction between Maternal Pre pregnancy Body Mass Index and Gestational Weight Gain Shapes Infant Growth. Acad Pediatr. 2014;14(5):463-470.

3. Abell S, Courten B, Boyle J, et al. Inflammatory and Other Biomarkers: Role in Pathophysiology and Prediction of Gestational Diabetes Mellitus. Int J Mol Sci. 2015;16(6):13442-13473.

4. Gavard J, Artal R. Effect of Exercise on Pregnancy Outcome. Clinical Obstetrics and Gynecology. 2008;51(2):467-480.

5. Bilbo S, Tsang V. Enduring consequences of maternal obesity for brain inflammation and behavior of offspring. The FASEB Journal. 2010;24(6):2104-2115.

6. Northstone K, Emmett PM, Rogers I. Dietary patterns in pregnancy and associations with nutrient intakes. Br J of Nutr. 2008;99(2):406-415.

7. McGowan CA, McAuliffe FM. Maternal dietary patterns and associated nutrient intakes during each trimester of pregnancy. Public Health Nutr. 2013;16(1):97-107.

8. Piirainen $\mathrm{T}$, Isolauri $\mathrm{E}$, Lagstro $\mathrm{H}$. Impact of dietary counseling on nutrient intake during pregnancy: a prospective cohort study. British Journal of Nutrition. 2006;96(6):1095-1104.

9. Choi J, Fukuoka Y, Lee J. The effects of physical activity and physical activity plus diet interventions on body weight in overweight or obese women who are pregnant or in postpartum: A systematic review and meta-analysis of randomized controlled trials. Preventive Medicine. 2013;56(6):351-364.
10. May LE, Suminski RR, Linklater ER, et al. Exercise during pregnancy: the role of obstetric providers. $J$ Am Osteopath Assoc. 2013;113(8):612619.

11. Mottola M, Giroux I, Gratton R, et al. Nutrition and Exercise Prevent Excess Weight Gain in Overweight Pregnant Women. Med Sci Sports Exer. 2010;42(2):265-272.

12. Boghossian N, Yeung E, Lipsky L, et al. Dietary patterns in association with postpartum weight retention. Am J Clin Nutr. 2013;97(6):13381345.

13. Subar A, Thompson F, Kipnis V, et al. Comparative Validation of the Block, Willett, and National Cancer Institute Food Frequency Questionnaires: The Eating at America's Table Study. Am J of Epidemiol. 2001;154(12):1089-1099.

14. Jukic A, Lawlor D, Juhl M, et al. Physical Activity during Pregnancy and Language Development in the Offspring. Paediatr Perinat Epidemiol. 2014;27(3):283-293.

15. Kaiser L, Campbell C. Practice Paper of the Academy of Nutrition and Dietetics Abstract: Nutrition and Lifestyle for a Healthy Pregnancy Outcome. J Acad Nutri Diet. 2014;114(9):1447.

16. Weng X, Odouli R, Li D. Maternal Caffeine Consumption during Pregnancy and the Risk of Miscarriage: A Prospective Cohort Study. Obstetric Anesthesia Digest. 2008;198:229-230.

17. Jersey S, Nicholson J, Callaway L, et al. An observational study of nutrition and physical activity behaviours, knowledge, and advice in pregnancy. BMC Pregnancy and Childbirth BMC Pregnancy Childbirth. $2013 ; 13: 115$. 